\title{
高等植物重金属耐性与超积累特性及其分子机理研究
}

\author{
孙瑞莲 $^{2} \quad$ 周启星 $^{1 *}$ \\ （1 中国科学院沈阳应用生态研究所陆地生态过程重点实验室, 沈阳 110016） \\ (2 中国科学院研究生院, 北京 100039)
}

\begin{abstract}
摘 要 由于重金属污染日益严重,重金属在土壤-植物系统中的行为引起了人们的高度重视。高等植物对重金 属的耐性与积累性, 已经成为污染生态学研究的热点。近年来, 由于分子生态学等学科的发展, 有关植物对重金属 的解毒和耐性机理、重金属离子富集机制的研究取得了较大进展。高等植物对重金属的耐性和积累在种间和基因 型之间存在很大差异。根系是重金属等土壤污染物进入植物的门户。根系分泌物改变重金属的生物有效性和毒 性, 并在植物吸收重金属的过程中发挥重要作用。土壤中的大部分重金属离子都是通过金属转运蛋白进入根细 胞, 并在植物体内进一步转运至液泡咜存。在重金属胁迫条件下植物螯合肽 (PC) 的合成是植物对胁迫的一种适应 性反应。耐性基因型合成较多的 PC, 谷胱甘肽 (GSH) 是合成 PC 的前体, 重金属与 PC 鳌合并转移至液泡中咜存, 从 而达到解毒效果。金属硫蛋白 (MTs) 与 PC一样, 可以与重金属离子螯合, 从而降低重金属离子的毒性。该文从分 子水平上论述了根系分泌物、金属转运蛋白、MTs、PC、GSH 在重金属耐性及超积累性中的作用, 评述了近 10 年来这 方面的研究进展, 并在此基础上提出存在的问题和今后研究的重点。
\end{abstract}

关键词 高等植物 重金属 耐性 超积累特性 植物修复

\section{HEAVY METAL TOLERANCE AND HYPERACCUMULATION OF HIGHER PLANTS AND THEIR MOLECULAR MECHANISMS: A REVIEW}

\author{
SUN Rui-Lian ${ }^{2}$ and ZHOU Qi-Xing ${ }^{1}$ * \\ (1 Key Laboratory of Terrestrial Ecological Process, Institute of Applied Ecology, Chinese Academy of Sciences, Shenyang 110016, China) \\ (2 Graduate School of Chinese Academy of Sciences, Beijing 100039, China)
}

\begin{abstract}
Owing to serious heavy metal pollution, much attention has been paid to its effects on soil-plant systems. The research of heavy metal tolerance and hyperaccumulation of higher plants has become a hot topic in the field of pollution ecology. With the development of molecular ecology, research on the mechanisms of heavy metal tolerance, detoxification and accumulation in higher plants has made progress in recent years. There are significant differences in the tolerance to and accumulation of heavy metals among higher plant species and genotypes. Root systems are the first entrance of heavy metal pollutants from the soil into plant. Root exudates reduce the availability and toxicity of metal pollutants and play an important role in ability for plants to absorb heavy metals. Almost all heavy metal ions enter root cells with the help of a metal transporter protein that are subsequently transported to the vacuole. The synthesis of PC in response to the stress caused by heavy metals is one of the adaptive responses common in higher plants. Heavy metal tolerant genotypes have higher levels of PC than non-tolerant genotypes under heavy metal stress. GSH is the substrate that synthesizes PC, which chelates the heavy metals. Heavy metal-PC chelatins are subsequently transported from the cytosol to the vacuole and heavy metal detoxification is thus achieved. MTs play the same role and in the same way as PC under heavy metal stress. The article reviews recent advances in understanding the role of root exudates, metal transporter proteins (MTs, PC and GSH), molecular mechanisms of heavy metal tolerance and hyperaccumulation in higher plants at the molecular level. Existing problems and major topics of future research were discussed.
\end{abstract}

Key words Higher plant, Heavy metal, Tolerance, Hyperaccumulation, Phytoremediation 
业生产所产生的“三废”的不合理排放, 导致土壤中 重金属含量急剧增加, 土壤-植物系统中重金属污染 问题日趋严重 (周启星, 1995; 孙铁珩等, 2001)。尽 管大多数高等植物在重金属污染的环境中不能生 存, 但人们发现仍有一些植物在较高浓度的重金属 污染环境中可以生长, 并且其中一些植物能积累超 寻常高含量的重金属(周启星和宋玉芳, 2004), 远远 超过了土壤中重金属含量。植物对重金属的耐性和 积累在品种间和种内存在明显的差异 (周启星等, 2003; 魏树和等, 2003)。Baker(1981)根据植物对重 金属的吸收、转移和积累机制将植物分为 3 类: 积累 型(超积累型)、指示型(敏感型)和排斥型。超积累 植物是指对重金属的吸收量超过一般植物 100 倍以 上的植物, 积累的 Cr、 $\mathrm{Co} 、 \mathrm{Ni} 、 \mathrm{Cu} 、 \mathrm{~Pb}$ 的含量一般在 $1.0 \mathrm{mg} \cdot \mathrm{kg}^{-1}$ (Dry weight, $D W$ ) 以上, 积累的 $\mathrm{Mn} 、 \mathrm{Zn}$ 含量一般在 $10 \mathrm{mg} \cdot \mathrm{kg}^{-1}(D W)$ 以上( 周启星和宋玉 芳, 2004）。大多数的超积累植物都生长在金属富集 的土壤上, 同时具有金属耐性的特征 (Bert \& Macnair, 2000), 但是对于一些超积累植物, 例如 Arabidopsis halleri 和 Thlaspi caerulescens 在正常土壤也有 分布并具有金属耐性,对于这些种类,许多学者认为 其积累性和耐性是不相关或相反的特性 (Meerts \& van Isacker, 1997)。Macnair 和 Bert(1999)认为, Arabidopsis halleri 对 $\mathrm{Zn}$ 的耐性和超积累性是两个相互 独立的遗传特性。Bert 和 Meerts (2003) 认为, Arabidopsis halleri 对 $\mathrm{Cd}$ 的耐性和积累性也是由独立遗 传控制的。这些研究暗示了超积累特性与耐性至少 在一定程度上是由不同基因控制的。相反, Lombi 和 Zhao(2000)研究 Thlaspi caerulescens 的结果显示其 对 Cd 的耐性和积累性是相互有关联的, 虽然缺乏确 丵的遗传证据, 但他们仍提出 $\mathrm{Cd}$ 超耐性和超积累性 以某种方式相联系。到目前为止, 植物对金属耐性 及超积累性之间的关系在分子水平上仍不完全清 楚。

一些普通植物在金属胁迫下的生理和分子机理 的研究已在进展中, 这些植物金属耐性基因的鉴定 和功能描述对于污染土壤的植物修复研究将是非常 有意义的。由于目前所发现的大部分超积累植物生 长速率慢, 生物量少, 限制了它们用于大规模污染土 壤修复的潜力, 因此阐明植物耐性、超积累性机制, 分离克隆相关基因, 利用植物基因工程技术, 获得高 效去除环境中污染物的转基因植物, 可能是培育优 良重金属超积累型植物的经济有效途径(周启星和 宋玉芳, 2004)。目前, 对植物重金属耐性、超积累特
性及其分子机理的研究, 已成为植物生态学和污染 生态学领域的前沿和热点。

\section{1 植物根系分泌物的功能及其分子生态学 基础}

根系分泌物是植物根系释放到周围环境中的各 种物质的总称, 其组成包括碳水化合物、氨基酸和有 机酸等。根分泌物的组成和含量变化是植物响应环 境胁迫最直接、最明显的反应之一(沈宏和严小龙, 2001)。根系分泌物在重金属污染的土壤中可以改 变重金属的化学行为与生态行为, 从而改变重金属 的有效性和对植物的毒性。一方面, 根系分泌物与 根际中某些游离的重金属离子螯合形成稳定的金属 螯合物复合体, 以降低其活度, 从而降低土壤中重金 属的移动性, 同时根系分泌物可以吸附、包埋重金属 污染物使其在根外沉淀下来, 而根系分泌的粘胶状 物质 (主要成分为多糖) 可与 $\mathrm{Pb}^{2+} 、 \mathrm{Cu}^{2+} 、 \mathrm{Cd}^{2+}$ 等重 金属离子竞争性结合使其滞留于根外, 这些都是植 物重金属耐性机制的体现; 另一方面, 根系分泌物中 大量的有机酸和酚类化合物与某些重金属如 $\mathrm{Fe}^{3+}$ 、 $\mathrm{Mn}^{2+} 、 \mathrm{Zn}^{2+}$ 形成络合物, 增加了这些重金属作为养 分离子的有效性, 有利于植物吸收利用, 增强植物抗 环境胁迫的能力, 这表现为植物对重金属的积累性。 目前有机酸对重金属的络合作用已成为根系分泌物 研究中的热点。

研究表明, 根系分泌物的主要成分为低分子量 有机酸 (Low-molecular-weight organic acids, LMWOA) 在土壤中金属离子的可溶性和有效性方面扮演着重 要角色, 如许多种类的低分子量有机酸均能影响土 壤固相结合 Cd 的释放, 形成 Cd-LMWOA 复合物, 增 加土壤 Cd 的溶解性 (Romheld \& Awad, 2000)。Cieslinski 等(1998) 通过对不同品种硬质小麦的根际土 的研究发现, 根际土壤中存在多种水溶性低分子量 有机酸, 其中乙酸和丁二酸占较大比例, 而非根际土 中则未检测到。高 Cd 积累品种 (Kyle) 根际土中低 分子量有机酸的总量明显高于低积累品种 (Arcola), 植物中 $\mathrm{Cd}$ 的积累量与根际土中低分子量有机酸的 含量成正比, 这表明植物的根系分泌物与植物体内 $\mathrm{Cd}$ 的积累可能有内在联系, 低分子量有机酸在土壤 中 $\mathrm{Cd}$ 的溶解性及植物吸收积累方面担负着重要作 用, 而且这种根系分泌物的差异性可能由一些特定 基因控制, 但目前还没有这方面的直接证据。与以 往研究不同, Zhao 等(2001)在 Zn 和 Cd 超积累植物 Thlaspi caerulescens 根系分泌物的研究中发现, $T$. 
caerulescens 的根系分泌物不能显著增强 $\mathrm{Zn}$ 和 $\mathrm{Cd}$ 的 移动性, 植物对 $\mathrm{Zn}$ 和 $\mathrm{Cd}$ 的超积累性与根系分泌物 无关。由于目前有关超积累植物根系分泌作用的研 究仍较少, 对于超积累植物是否可能分泌某些特殊 有机物, 促进土壤中金属溶解和根际的吸收, 还缺乏 直接的证据。

在植物正常生长发育过程中, 根系分泌物大多 是植物次生代谢产物, 很可能表现为数量遗传的微 效基因控制, 而特异性根分泌物的组成、含量则受胁 迫条件的影响, 是一个重要的遗传性状。对特定胁 迫条件下植物根系产生特定分泌物的生理及分子机 理的研究,有利于揭示植物适应环境胁迫的实质。 尽管目前关于环境胁迫与植物根分泌物的关系有许 多报道, 然而有关环境胁迫诱导特定根分泌物的分 泌作用及相关基因的定位和克隆还很少报道。麦根 酸类植物铁载体的基因定位、克隆是植物根分泌物 基因水平研究上较为成功的一个例子 (Okumura $e t$ $a l ., 1994)$ 。该研究表明, 麦根酸类植物铁载体的分 泌只受 $52 \mathrm{kD}$ 和 $53 \mathrm{kD}$ 两条多肽的控制, 人们已经能 检测到由缺铁诱导的特异性 $\mathrm{cDNA}(I d s 1, I d s 2, \cdots$ $I d s 7$ ), 并对克隆到的 $I d s 1 、 I d s 2 、 I d s 3$ 的序列进行了 分析, Ids 基因的发现使进一步研究铁载体生物合成 成为可能。铝毒胁迫时, 菾麦抗铝品种靠大量分泌 草酸以及体内累积草酸来解除铝毒害作用, 麻密等 (2000) 在研究小麦耐铝毒性时发现,位于 $3 R$ 染色体 短臂上的耐铝基因与有机酸分泌有密切关系。Basu 等(1997)在小麦( Triticum aestivum)对铝抗性研究中 发现, 在铝 $(\mathrm{Al})$ 胁迫条件下, 耐 $\mathrm{Al}$ 植物根系分泌一 系列特异的低分子量多肽进入根际环境, 其中 $23 \mathrm{kD}$ 多肽表现出对 $\mathrm{Al}$ 强烈的结合作用,但对于进一步鉴 定并克隆编码 $23 \mathrm{kD}$ 多肽基因的研究还未见报道。

\section{2 金属转运蛋白的功能及其分子生态学基} 础

土壤中的重金属离子进入植物体内的第一步是 根系吸收, 无论是普通植物或是超积累植物, 大部分 金属离子都是通过专一或通用的离子载体(如转运 蛋白)进入根细胞的。Lasat 等(1996)通过比较超积 累植物 Thlaspi caerulescens 和非超积累植物 T. arvense 对 $\mathrm{Zn}^{2+}$ 的吸收动力学, 研究发现两者的根系在 $\mathrm{Zn}^{2+}$ 吸收的饱和吸附阶段具有相似的米氏常数 $(\mathrm{Km})$, 但两者的最大吸收速率 $\left(V_{\max }\right)$ 差异明显, 前 者是后者的 4.5 倍, 进一步发现两种植物对 $\mathrm{Zn}^{2+}$ 具
有相似的亲和力, 不同的是 T. caerulescens 在单位鲜 重的根系细胞膜上分布更多的 $\mathrm{Zn}^{2}+$ 转运蛋白, 从而 使植株具有对 Zn 的超积累特性。Küpper 等(1999) 研究还表明, 跨膜的金属转运蛋白在重金属的吸收、 木质部的装载与卸载以及液泡区室化作用中, 可能 起着决定性作用。此外, 超积累植物对重金属的吸 收有很强的选择性, 只吸收和积累生长介质中一种 或几种特异性金属 (Salt \& Krämer, 2000)。解释这 种选择性积累的可能机制是: 在金属跨根细胞进入 根细胞共质体或跨木质部薄壁细胞的质膜装载进入 木质部导管时, 由专一性转运蛋白或通道蛋白调控。

近年来, 人们在分子水平上对植物微量元素的 转运机制做了许多研究, 在植物以及真菌、动物中克 隆到了多个编码微量元素转运蛋白的基因, 其中 ZIP 家族 (ZRT, IRT-like protein) 和 CDF 家族 (Cation diffusion facilitator) 是研究较多的基因家族。ZIP 基 因家族编码的蛋白一般具有 8 个跨膜区, 而 CDF 仅 含 6 个跨膜区。据最新资料统计, 目前 ZIP 基因家 族的成员已经上升到 86 个, $\mathrm{CDF}$ 基因家族成员则升 至 101 个, 它们在重金属的运输过程中担负着重要 作用 (Gaither \& Eide，2001)。ZRT1、ZRT2（Zn 吸收 运输蛋白基因)和 $I R T 1$ ( $\mathrm{Fe}$ 运输蛋白基因)是最早克 隆到的 ZIP 基因家族成员。有研究证实,由 ZIP 基 因编码蛋白与酵母中 $Z R T 1$ 和 $Z R T 2$ 基因编码蛋白、 拟南芥中的 IRT 1 基因编码蛋白有高度的同源性 (Zhao \& Eide, 1996a, 1996b; Eide \& Broderius, 1996)。Pence 等 (2000) 在超积累植物 Thlaspi caerulescens 中克隆到了 ZNT1 基因, 属于 ZIP 基因家 族并与拟南芥 (Arabidopsis) 中的 ZIP4 基因高度同 源。在缺 $\mathrm{Zn}$ 和 $\mathrm{Zn}$ 供应充足的条件下, $Z N T 1$ 可以在 Thlaspi caerulescens 的根系和叶片中高量表达; 而对 于非超积累植物 Thlaspi arvense, ZNT1 只在缺 $\mathrm{Zn}$ 条 件下表达, Zn 供应充足时, 表达明显受到抑制。可 见, ZNT1 在 Thlaspi caerulescens 的高量表达与 $\mathrm{Zn}$ 的 有效性无关, 这可能是它超吸收 $\mathrm{Zn}$ 的主要原因。 Assunção 等(2001) 从超积累植物 Thlaspi caerulescens 中分离并克隆了 3 个 $\mathrm{Zn}$ 转运蛋白基因, 分别是 $Z I P 1 、 Z N T 1 、 Z N T 2$ 基因; 他们研究发现, 3 个 $\mathrm{Zn}$ 转 运蛋白基因在 Thlaspi caerulescens 中的表达量要明显 高于 Thlaspi arvense, 根系中 $\mathrm{Zn}$ 转运蛋白基因的表达 量与 Thlaspi caerulescens 富集 Zn 的能力成正相关; 并 且认为, ZIP1 的过量表达可能有助于提高植株对 $\mathrm{Zn}$ 的耐性。

Conklin 等(1992)在酵母 Saccharomyces cerevisiae 
中发现了 $\mathrm{CDF}$ 家族的两个成员, $Z r c 1$ 和 $\operatorname{Cot} 1$ 基因。 当 $Z r c 1$ 或 $\operatorname{Cot} 1$ 基因过量表达时, 细胞耐 $\mathrm{Zn}$ 或耐 $\mathrm{Zn}$ 和 $\mathrm{Co}$ 的能力增强; 而当 $Z r c 1$ 或 $\operatorname{Cot} 1$ 基因发生突变 时, 则导致细胞对 $\mathrm{Zn}$ 高度敏感。 $Z r c 1$ 和 $\operatorname{Cot} 1$ 蛋白 是 Zn 区室化相关蛋白, 可以把 $\mathrm{Zn}$ 运输到液泡中, 从 而起到解毒的作用。CDF 家族成员在植物中有较多 分布, 仅拟南芥中就有 10 个基因家族成员 (Gaither \& Eide, 2001)。到目前为止, 在植物中, 对于 CDF 家 族成员的研究大多集中在拟南芥中的 ZAT 基因。 Zaal 等(1999)从拟南芥中分离到了 $Z A T$ 基因, 它与 哺乳动物的 Zn 转运蛋白基因 ZNT2、ZNT3 和 ZNT4 有高度同源性, 同源性达 35\% 40\%。在高 Zn 浓度 下,当 $Z A T$ 基因在拟南芥中过量表达时, 植株的耐 $\mathrm{Zn}$ 能力增强, 同时根中 $\mathrm{Zn}$ 的含量增加, 这表明 $Z A T$ 蛋白可能与植物细胞重金属区室化有关。Persans 等(2001)报道从镍超积累植物 Thlaspi goesingense 中 分离到的 $\mathrm{CDF}$ 家族成员 $T g M T P 1$ 属于液泡转运蛋 白基因。

另一类与金属离子吸收有关的蛋白是 Nramp 家 族 (Natural resistance associated macrophage proteins)。 与 ZIP 和 CDF 家族不同, Nramp 基因家族编码的蛋 白一般具有 12 个跨膜区。Nramp 1 最初在哺乳动物 中发现, 是该家族中被鉴定的第一个成员。在小鼠 中, 这个基因控制了巨噬细胞和单核细胞对无关的 抗原性的寄生物在细胞内增殖的限制, 被任命为天 然抵抗力相关的巨噬细胞蛋白 (Belouchi et al., 1995)。植物中的研究主要集中在水稻 Oryza sativa 和拟南芥。例如 Abdelmajid 等 (1997) 从水稻 (Oryza sativa) 中分离并克隆了 $O s N r a m p 1 、 O s N r a m p 2$ 和 $O s$ Nramp3 基因。Supek 等( 1996) 在酵母中发现了一个 Nramp 家族成员-SMF1, 它编码 Mn 转运蛋白, 这个 发现使 Nramp 家族编码植物金属转运蛋白成为可 能。目前关于 Nramp 基因家族在高等植物中的功能 仍不明确, Nramp 基因与金属离子吸收的关系有待 于更进一步的研究。

\section{3 金属硫蛋白的功能及其分子生态学基础}

金属硫蛋白 (Metallothioneins, MTs) 是自然界中 普遍存在的低分子量、富含半胱氨酸的多肽 (Kagi \& Schaffer, 1988)。目前所发现的 MTs 可分为 3 类。 哺乳动物的金属硫蛋白通常由 61 个氨基酸残基组 成, 分子量为 $6 \sim 7 \mathrm{kD}$, 所含 20 个 Cys 残基分布相对 固定。这种一级结构很相似的金属硫蛋白称为 MTI。MT- II 亦为富含半胱氨酸的低分子量蛋白, 但
其 Cys 残基的分布与 MT- I 不尽相同。MT- I 和 MTII 都是由基因直接转录合成。MT- III 是一类结构特 殊的多肽, 亦称为植物螯合肽 (Phytochelatin, PC), 结 构多为 $\left(\gamma\right.$-Glu-Cys ${ }_{\mathrm{n}}$-Gly, $n=2 \sim 11$ 。 PC 不是由基因 直接编码, 必须在 PC 合酶的催化下完成(麻密等, 2000)。Nathalie 等(2001)研究表明, MTs 能够通过硫 基与金属离子结合, 从而降低重金属离子的毒性, 它 在植物对 $\mathrm{Zn}^{2+}$ 和 $\mathrm{Cu}^{2+}$ 的解毒过程中起着重要作用。

小麦 $\mathrm{Ec}$ 蛋白是植物中最先分离得到纯化蛋白 质的 MT- II (Lane et al. , 1987), 其 Cys 残基的分布 主要为 Cys-Xaa-Cys 形式, 具有结合金属离子的能 力。尽管目前在高等植物中分离到的具有 Cys-XaaCys 典型结构的 MT 只有小麦 Ec 蛋白一例, 但编码 这类蛋白的基因已在多种高等植物中发现, 许多高 等植物 MT(或 MT-like) 基因已在 GenBank 注册并公 布。但这些基因的翻译产物尚未从植物组织中分 离, 研究证明 MT-like 蛋白中间区的水解可能正是在 高等植物中分离完整的 MT-like 蛋白难以成功的原 因( Robinson et al., 1993)。根据 Cys 残基位置的不 同, 高等植物 MT-like 基因推测产物 (MT-like 蛋白) 可分为 1 型和 2 型, 1 型中 Cys 残基的唯一形式为 Cys-Xaa-Cys, 而 2 型的 N-末端结构域 Cys 残基有 3 种形式: Cys-Cys、Cys-Xaa-Cys 和 Cys-Xaa-Xaa-Cys。

Robinson 等(1993) 早在 1993 年就指出, 1 型基 因在植物根中转录水平较高, 如大麦 (Hordeum vulgare)、玉米( Zea mays) 中的 MT-like 基因, 而 2 型基 因在叶中的转录水平明显高于根中, 如菜豆 (Phaseolus vulgaris)、蓖麻( Ricinus communis) 中的 MT-like 基 因。拟南芥中 $M T 1 、 M T 2$ 基因分属于 1 型和 2 型, 其中 $M T 1$ 基因主要在根和种子中表达, MT2 主要在 叶子中。这两种基因在重金属 $\mathrm{Cd}^{2}+$ 和 $\mathrm{Cu}^{2+}$ 的胁迫 下 mRNA 的积累均明显增加。Murphy 等(1997)研究 发现, MT2 在拟南芥中的表达受 $\mathrm{Cu}^{2+}$ 的诱导, 并且 表达强度与不同基因型抗铜胁迫的能力密切相关。 liasegawa 等(1997)将酵母 $M T$ 基因( CUP1)转入花椰 菜( Brassica oleracea var. botrytis) 中发现, 转基因的花 椰菜对 Cd 的耐性和积累性都要高于未转基因的植 株, 尤其是在上部的叶片中。 $\mathrm{Ma}$ 等(2003)在重金属 耐性植物紫羊茅( Festuca rubra cv. Merlin) 中克隆到 $m c M T 1$ 基因的全长 $c D N A$, 测序后证实此基因属于 1 型。将 $m c M T 1$ 基因转入酵母 MT 基因缺失突变株 中发现, $m c M T 1$ 的表达增加了酵母细胞对重金属 $\mathrm{Cd}^{2+} 、 \mathrm{Cu}^{2+} 、 \mathrm{Zn}^{2+} 、 \mathrm{~Pb}^{2+}$ 和 $\mathrm{Cr}^{2+}$ 的抗性, 研究证实了 $m c M T 1$ 基因的表达产物具有抗重金属的功能。目 
前尽管已经证实 MT 基因存在于许多种植物中, 但 大多数植物对重金属都不表现耐性, 它们在植物中 的表达产物和功能仍然不清楚。对于 MTs 是否是 植物高耐重金属的主要机制仍不明确。

\section{4 植物螯合肽的功能及其分子生态学基础}

Grill 等 (1985) 首次在 Cd 胁迫蛇根木 (Rauvolfia serpentina $)$ 的植物细胞中分离得到植物螯合肽 (Phytochelatin, $P C$ )。研究证实, $\mathrm{PC}$ 是一种由半胱氨酸、 谷氨酸和甘氨酸组成的含巯基螯合多肽, 分子量一 般为 $1 \sim 4 \mathrm{kD}$, 结构多为 $(\gamma \text {-Glu-Cys })_{\mathrm{n}}$-Gly, $\mathrm{n}=2 \sim 11$ 。 由于其巯基含量高, 对重金属的亲和力大, 能够螯合 多种重金属离子,使重金属离子失去活性。PC 的发 现, 使人们对植物重金属耐性机理产生了新的认识, 认为 $\mathrm{PC}$ 的形成才是植物解毒的重要生理机制。到 目前为止, 在蛇根木 (Rauwolfia serpentina)、烟叶 (Nicotiana tabacum)、毛曼陀罗 (Datura innoxia)、菜 豆、番茄 ( Lycopersicon esculentum)、甘蓝( Brassica oleracea )、玉米和小麦等植物中均发现 PC 的存在。在 高等植物中分离得到的 PC 大多数为镉离子结合 物。 $\mathrm{PC}$ 与 $\mathrm{Cd}$ 的络合物可以被分为低分子量 (LMW) 和高分子量 ( HMW ) 复合物两类 ( Rauser，1995)。 LMW 复合物是 $\mathrm{Cd}^{2+}$ 从细胞质向液泡中转运的主要 形式, $\mathrm{HMW}$ 复合物是 $\mathrm{Cd}^{2+}$ 在液泡中积累的主要形 式。 HMW 对 $\mathrm{Cd}^{2+}$ 的结合能力大于 LMW, 因而在液 泡中 HMW 复合物比较稳定。 PC 与 $\mathrm{Cd}$ 之间的特殊 关系还在于 $\mathrm{Cd}^{2+}$ 诱导 $\mathrm{PC}$ 及 $\mathrm{PC}$ 合酶的能力很强。 Grill 等(1987)报道,在 10 多种高等植物中 $\mathrm{Cd}^{2}+$ 诱导 $\mathrm{PC}$ 的作用最强, 其它金属依次为 $\mathrm{Pb}^{2+}>\mathrm{Zn}^{2+}>\mathrm{Sb}^{3+}$ $>\mathrm{Ag}^{+}>\mathrm{Hg}^{2+}>\mathrm{As}^{5}->\mathrm{Cu}^{2+}>\mathrm{Sn}^{2+}>\mathrm{Au}^{3+}>\mathrm{Bi}^{3+}$ 。 Grill 等(1989)首次在合成 PC 的细胞中发现了 PC 合 酶 ( $\gamma$-谷胺酰半胱氨酸二肽酶), 该酶在重金属离子 的激发下,催化形成 PC, 而谷胱甘肽 ( GSH) 是 PC 合 成的前体。Knecht 和 Dillen（1994）、Klapheck 等 （1995）也相继在高等植物中发现重金属诱导的 PC 合酶。重金属诱导效率依次为 $\mathrm{Cd}^{2+}>\mathrm{Ag}^{+}>\mathrm{Pb}^{2+}>$ $\mathrm{Cu}^{2+}>\mathrm{Hg}^{2+}>\mathrm{Zn}^{2+}>\mathrm{Sn}^{2+}>\mathrm{Au}^{3+}>\mathrm{As}^{5}->\mathrm{In}^{3+}>$ $\mathrm{Tl}^{3+}>\mathrm{Ge}^{4+}>\mathrm{Bi}^{3+}>\mathrm{Ga}^{3+}$ 。此外, 具有 $(\gamma-\mathrm{Glu}-\mathrm{Cys})_{\mathrm{n}^{-}}$ $\beta-A l a$ 结构的肽链也在菜豆属植物中被发现, 经检测 证实此物质为丙氨酸类 $\mathrm{PC}$ (Grill et al.，1986), 类 PC 可参与植物的重金属解毒作用, 在一定条件下, 植物可同时合成 PC 和类 PC, 并协同解毒。

Steffens 等(1986)研究表明, 在高浓度 $\mathrm{Cd}^{2+}$ 培养 液中, 耐 $\mathrm{Cd}$ 番茄植株大量积累 PC, 而 Cd 敏感品种
却无此现象, 如在培养液中加入 GSH 合酶抑制剂 $\mathrm{BSO}, \mathrm{PC}$ 合成量大大减少, 耐 $\mathrm{Cd}$ 品种不再表现原有 的耐性, 加入适量 GSH 可解除 BOS 的抑制作用。 Kneer 和 Zenk (1992)发现, 在 $\mathrm{Cd}^{2+}$ 胁迫下耐 $\mathrm{Cd}$ 植物 体内 $\mathrm{Cd}^{2+}$-PC 含量比对照高 $10 \sim 1000$ 倍。Zenk （1996）的进一步研究表明, 重金属离子经细胞壁和 细胞膜进入细胞质, 激活 PC 合酶, 在胞质内以 $\mathrm{GSH}$ 为底物酶促合成 PC, PC 螯合重金属离子, 形成重金 属-PC 螯合物, 从而使重金属离子失去活性。重金 属-PC 鳌合物可在 ATP 的作用下通过液泡膜转运至 液泡, 可见 $\mathrm{PC}$ 在重金属离子区室化中起着重要作 用。目前关于 PC 的研究大多集中于耐性植物或普 通植物, 已经成为植物抗重金属胁迫的研究热点之 一,而对于超积累植物研究的相对较少。Ebbs 等 （2002）曾报道在 $\mathrm{Cd}^{2+}$ 胁迫下, 超积累植物 Thlaspi caerulescens 和非超积累植物 Thlaspi arvense 中都有 PC 的合成, 但 Thlaspi caerulescens 中 PC 量明显少于 Thlaspi arvense, 看来 PC 与 Thlaspi caerulescens 对 $\mathrm{Cd}$ 的积累无关, 这在一定程度上表明 Thlaspi caerulescens 中可能存在着其它的积累机理。PC 在超 积累植物中是否起到重要作用仍有待于进一步研 究。

近年来, 许多学者相继从植物、酵母中分离克隆 到编码 PC 合酶的基因。Clemens 等(1999) 从小麦 $\mathrm{cDNA}$ 分离克隆到 $T a P C S 1$ 基因, 该基因在酵母 $S a c-$ charomyces cerevisiae 中表达对 $\mathrm{Cd}$ 的高耐性。 $\mathrm{Ha}$ 等 (1999)从 Cd-敏感拟南芥突变体 Cad1-1( Arabidopsis thaliana)、裂殖酵母属 (Schizosaccharomyces pombe) 中 分离克隆到同源基因 $A t P C S 1 、 S p P C S$, 具有耐重金属 的特性, 在 $\mathrm{Cd}^{2+} 、 \mathrm{Cu}^{2+}$ 胁迫下, PCS 基因缺失的裂殖 酵母不能合成 $\mathrm{PC}$, 对 $\mathrm{Cd}^{2+} 、 \mathrm{Cu}^{2+}$ 表现高度敏感性。 当给 PCS 基因缺失的裂殖酵母再接种纯化酵母 PCS 蛋白时, 即可激活其 PC 合酶活性, 表现出耐重金属 特性。

由于 $\mathrm{GSH}$ 与 $\mathrm{PC}$ 之间特殊的关系, 而 $\mathrm{GSH}$ 的合 成又依赖于半胱氨酸活性, 因此对于 GSH 和半胱氨 酸合成和代谢的分子机理研究也成为热点问题之 一。Xiang 和 Oliver( 1998) 研究发现, GSH 是以半胱 氨酸为底物, 通过两步酶促反应完成, $\gamma$-ECS 合成酶 和 GSH 合成酶是其中的关键酶, 编码这两个酶的分 别为 $\gamma$-ECS 基因 ( $g s h 1)$ 和 $g s h 2$ 基因。Noctor 等 (1998)研究发现, 在 $\mathrm{Cd}^{2+}$ 胁迫下, $g s h 1$ 基因的高度 表达, 可显著提高白杨树叶中 GSH 含量, 大量合成 $\mathrm{PC}$, 在植株体内以 Cd-PC 螯合物的形式大量积累重 
金属, 解除重金属对植株的毒害作用。Zhu 等 （1999）报道, 将 $g s h 1$ 基因与 gsh2 基因分别转入印 度芥菜幼苗中, 发现转基因印度芥菜对 $\mathrm{Cd}^{2}+$ 的耐性 和积累能力明显增加, 并且其耐性和积累能力与 $g s h 2$ 基因的表达成正相关。Cintia 等(2004)研究发 现, 在 $\mathrm{Cd}^{2+} 、 \mathrm{Se}^{3+} 、 \mathrm{Ni}^{2+}$ 的介质中, 半胱氨酸合酶基 因过量表达的转基因烟草中 GSH 和半胱氨酸含量 增加, 对重金属的耐性要明显高于野生型烟草, 并且 与其它类型的转基因植株相比, 在液泡和叶绿体中 半胱氨酸合酶基因均过量表达的转基因植株对重金 属表现出更高的耐性, 并且可以增加 Cd 在植株地上 部的积累。

\section{5 问题与展望}

随着生物技术的发展及其在生态学研究中的应 用, 使人们从分子水平上阐明植物对重金属离子的 吸收、积累和忍耐机理成为可能。近年来, 环境胁迫 植物耐性基因和超积累基因的分子生态学研究已经 开始, 并取得了一定进展, 但对于这些基因所编码蛋 白的功能和调控机理, 多数仍缺乏足够的了解。在 分子水平上,植物对重金属耐性和积累性之间的关 系仍不明确。根系分泌物是否在超积累植物根系吸 收重金属离子的过程中担负重要作用? 与植物耐重 金属有关的基因是如何表达和调控的? 不同金属转 运蛋白之间的相互关系和调控途径如何? 植物中是 否可能还存在着尚未发现的其它耐性和超积累性机 制? 等等。这些问题有待于进一步探明。对于上述 问题的研究,一方面可为利用植物基因工程技术培 育低积累高耐性的农作物新品种, 提供理论和技术 基础; 另一方面, 可以阐明超积累植物耐性和超积累 机制, 分离克隆相关基因, 转入生长快且生物量大的 植物中, 从而培育出可以高效去除环境中重金属污 染物的植物, 为重金属污染土壤的修复提供理想的 材料。

\section{参 考 文 献}

Abdelmajid B, Tony K, Philippe G (1997) . Cloning and characterization of the OsNramp family from Oryza sativa, a new family of membrane proteins possibly implicated in the transport of metal ions. Plant Molecular Biology, 33,1085 - 1092.

Assunção AGL, Martins PDC, Folter SD (2001). Elevated expression of metal transporter genes in three accessions of the metal hyperaccumulator Thlaspi caerulescens. Plant, Cell and Environment, 24,217- 226 .

Baker AJM (1981). Accumulators and excluders - strategies in the response of plants to heavy metals. Journal of Plant Nutrition, $3,643-654$.

Basu U, McDonald-Stephens JL, Archambault DJ, Good AG, Briggs KG (1997). Genetic and physiological analysis of doubledhaploid, aluminium-resistant lines of wheat provide evidence for the involvement of a $23 \mathrm{kD}$, root exudate polypeptide in mediating resistance. Plant and Soil, 196,283-288.

Belouchi A, Cellier M, Gros P (1995). The macrophage-specific membrane protein Nramp controlling natural resistance to infections in mice has homologue express in the system of plant. Plant Molecular Biology, 9,1181 - 1196.

Bert V, Macnair MR (2000). Zinc tolerance and accumulation in metallicolous and non-metallicolous populations of Arabidopsis halleri (Brassicaceae). New Phytologist, 146,225-233.

Bert V, Meerts P (2003). Genetic basis of Cd tolerance and hyperaccumulation in Arabidopsis halleri. Plant and Soil, 249,918.

Cieslinski G, van Rees KCJ, Szmigielska AM, Krishnamurti GSR, Huang PM (1998). Low-molecular-weight organic acids in rhizosphere soils of durum wheat and their effect on cadmium bioaccumulation. Plant and Soil, 203,109-117.

Cintia GK, Masaaki N, Michimi N (2004). Heavy metal tolerance of transgenic tobacco plants over-expressing cysteine synthase. Biotechnology Letters, 26,153-157.

Clemens S, Kim EJ, Neumann D (1999). Tolerance to toxic metals by a gene family of phytochelatin synthases from plants and yeast. EMBO Journal, 18,3325-3333.

Conklin DS, McMaster JA, Culbertson MR (1992) . COT1, a gene involved in cobalt accumulation in Saccharomyces cerevisiae. Molecular Cell Biology, 12,3678-3688.

de Knecht JA, van Dillen M (1994). Phytochelatins in cadmiumsensitive and cadmium-tolerant Silene vulgaris. Plant Physiology, $104,255-261$.

Ebbs S, Lau I, Ahner B (2002). Phytochelatin synthesis is not responsible for Cd tolerance in the $\mathrm{Zn} / \mathrm{Cd}$ hyperaccumulator Thlaspi caerulescens. Planta, 214,635-640.

Eide D, Broderius M (1996). A novel iron-regulated metal transporter from plants identified by functional expression in yeast. Proceedings of the National Academy of Sciences, 93, 5624 5628 .

Gaither AL, Eide DJ (2001). Eukaryotic zinc transporters and their regulation. BioMetals, 14,251-270.

Grill E, Gekeler W, Winnacker EL, Zenk MH (1986). Homophytochelatins are heavy-metal-binding peptides of homo-GSH containing Fabales. FEBS Letters, 205,47-50.

Grill E, Löffler S, Winnacker EL, Zenk MH ( 1989). Phytochelatins, the heavy-metal-binding peptides of plants, are synthesized from glutathione by a specific $\gamma$-glutamycysteine dipeptidy1 transpeptidase (phytochelatin synthase). Proceedings of the National Academy of Sciences USA, 86,6838-6842.

Grill E, Winnacker EL, Zenk MH (1985). Phytochelatins: the 
principal heavy-metal complexing peptides of higher plants. Science, $230,674-676$.

Grill E, Winnacker EL, Zenk MH (1987). Phytochelatins: a class of heavy-metal-binding peptides from plants, are functionally analogous to metallothioneins. Proceedings of the National Academy of Sciences USA, 84,439-443.

Ha SB, Simith AP, Howden R (1999). Phytochelatin synthase genes from Arabidopsis and the yeast Schizosaccharomyces pombe. Plant Cell, 11,1153 - 1163 .

liasegawa I, Emiko T, Michio S (1997). Genetic improvement of heavy metal tolerance in plants by transfer of the yeast metallothionein gene (CUP1). Plant and Soil, 196,277-281 .

Kagi JHR, Schaffer A (1988). Biochemistry of metallothionein. Biochemistry, 27,8509-8551 .

Klapheck S, Schlunz S, Bergmann L (1995). Synthesis of phytochelatins and homo- phytochelatins in Pisum sativum L. Plant Physiology, 107,515 - 521 .

Kneer R, Zenk MH (1992). Phytochelatins protect plant enzymes from heavy metal poisoning. Phytochemistry, 31,2663- 2667.

Küpper H, Zhao FJ, McGrath SP (1999) . Cellular compartmentation of zinc in leaves of the hyperaccumulator Thlaspi caerulescens. Plant Physiology, 119,305-311.

Lane B, Kajioka R, Kennedy T (1987). The wheat germ Ec protein is a zinc-containing metallothionein. Biochemistry Cell Biology, $65,1001-1005$.

Lasat MM, Baker AJM, Kochian LV (1996) . Physiological characterization of root $\mathrm{Zn}^{2+}$ absorption and translocation to shoots in $\mathrm{Zn}$ hyperaccumulator and non-accumulator species of Thlaspi. Plant Physiology, 112,1715-1722.

Lombi E, Zhao FJ (2000). Cadmium accumulation in populations of Thlaspi caerulescens and Thlaspi goesingense. New Phytologist, $145,11-20$.

Ma M (麻密), Jia YT (贾燕涛), Huang YS (黄玉山) (2000). Molecular mechanisms of higher plant resistance to heavy metal. In: Lin ZP (林忠平) ed. Green Gene For 21 st Century (走向 21 世纪的植物分子生物学). Science Press, Beijing, $184-$ 192. (in Chinese)

Ma M, Lau PS, Jia YT (2003). The isolation and characterization of Type 1 metallothionein (MT) cDNA from a heavy-metal-tolerant plant, Festuca rubra cv. Merlin. Plant Science, 164,5160 .

Macnair MR, Bert V (1999). Zinc tolerance and hyperaccumulation are genetically independent characters. Proceedings of the Royal Society of London B, 266,2175 - 2179.

Meerts P, van Isacker N (1997). Heavy metal tolerance and accumulation in metallicolous and non-metallicolous populations of Thlaspi caerulescens from continental Europe. Plant Ecology, $133,221-231$.

Murphy A, Zhou J, Goldsbrough PB (1997). Purification and immunological identification of metallothioneins 1 and 2 from Arabidopsis thaliana. Plant Physiology, 113,1293-1301 .
Nathalie ALM, Hassinen VH, Hakvoort HWJ (2001). Enhanced copper tolerance in Silene vulgaris (Moench) garcke populations from copper mines is associated with increased transcript levels of a 2b-type metallothionein gene. Plant Physiology, 126,15191526 .

Noctor G, Arisi ACM, Jouanin L (1998). Manipulation of glutathione and amino acid biosynthesis in the chloroplast. Plant Physiology, 118,471 - 482 .

Okumura N, Nishizawa NK, Umehare Y ( 1994 ). Adioxygenase ( Ids2) expressed under iron deficiency conditions in the roots of Hordeum vulgare. Plant Molecular Biology, 25,705-719.

Pence NS, Larsen PB, Ebbs SD, Letham DLD, Lasat MM (2000). The molecular physiology of heavy metal transport in the $\mathrm{Zn} / \mathrm{Cd}$ hyperaccumulator Thlaspi caerulescens. Proceedings of the $\mathrm{Na}$ tional Academy of Sciences USA, 97,4956-4960.

Persans MW, Nieman K, Salt DE (2001). Functional activity and role cation-efflux family members in $\mathrm{Ni}$ hyperaccumulation in Thlaspi goesingens. Proceedings of the National Academy of Sciences USA, 98,9995 - 10000 .

Rauser WE (1995). Phytochelatins and related peptides. Plant Physiology, 109,1141-1149.

Robinson NJ, Evans IM, Cheeks C (1993). Plant metallothioneins. Biochemistry Journal, 295,1-10.

Romheld V, Awad F (2000). Significance of root exudates in acquisition of heavy metal from a contaminate calcareous soil by graminaceous species. Journal of plant Nutrition, 23, 1857 1866 .

Salt DE, Krämer U (2000). Mechanisms of metal hyperaccumulation in plants. In: Raskin H, Ensley BD eds. Phytoremediation of Toxic Metal: Using Plants to Clear up the Environment. John Wiley \& Sons, New York, 231 - 246.

Shen H (沈宏), Yan XL (严小龙) (2001). Root exudation and their induced mechanism. Soil and Environmental Sciences (土壤 与环境), 10,339-342. (in Chinese with English abstract)

Steffens JC, Hunt DF, Williams BG (1986). Accumulation of nonprotein metal-binding polypeptides $(\gamma \text {-Glu-Cys })_{\mathrm{n}}$-Gly in selected cadmium-resistant tomato cells. Journal of Biological Chemistry, $261,13879-13882$.

Sun TH (孙铁街), Zhou QX (周启星), Li PJ (李培军) (2001). Pollution Ecology (污染生态学). Science Press, Beijing. (in Chinese)

Supek F, Supekova L, Nelson H, Nelson N (1996). A yeast manganese transporter related to the macrophage protein involved in conferring resistance to mycobacteria. Proceedings of the National Academy of Sciences USA, 93,5105- 5110

van der Zaal EJ, Neuteboom LW, Pinas JE (1999). Overexpression of a zinc transporter gene from Arabidopsis can lead to enhanced zinc resistance and zinc accumulation. Plant Physiology, $119,1-9$.

Wei SH (魏树和), Zhou QX (周启星), Wang X (王新), Cao W (曹伟), Ren LP (任丽萍), Song YF (宋玉芳) (2003). 
Identification of weed species with hyperaccumulative characteristics of heavy metals. Progresses in Natural Science (自然科学进 展), 13,1259-1265. (in Chinese with English abstract)

Xiang C, Oliver D (1998). Glutathione metabolic genes coordinately respond to heavy metals and jasmonic acid in Arabidopsis. Plant Cell, 10,1539 - 1550 .

Zenk MH (1996). Heavy metal detoxification in higher plants. Gene, 179,21-30.

Zhao FJ, Hamon RE, Mclaughlin MJ (2001). Root exudates of the hyperaccumulator Thlaspi caerulescens do not enhance metal mobilization. New Phytologist, 151,613-620.

Zhao H, Eide D (1996a). The yeast ZRT1 gene encodes the zinc transporter protein of a high affinity uptake system induced by zinc limitation. Proceedings of the National Academy of Sciences, $93,2454-2458$.

Zhao H, Eide D (1996b). The ZRT2 gene encodes the low affinity zinc transporter in Saccharomyces cerevisiae. Journal of Biological
Chemistry, 271,23203-23210.

Zhou QX (周启星) (1995). Ecology of Combined Pollution(复合 污染生态学). China Environmental Science Press, Beijing. (in Chinese)

Zhou QX (周启星), Cheng Y (程云), Zhang QR (张倩茹), Liang JD (粱继东) (2003). Quantitative analyses of relationships between ecotoxicological effects and combined pollution. Science in China (中国科学), 33, 566-573. (in Chinese with English abstract)

Zhou QX (周启星), Song YF (宋玉芳) (2004). Remediation of Contaminated Soils: Principles and Methods (污染土壤修复原 理与方法). Science Press, Beijing. (in Chinese)

Zhu YL, Pilon-Smits EAH, Tarun AS (1999). Cadmium tolerance and accumulation in Indian mustard is enhanced by overexpressing $\gamma$-glutamycysteine synthetase. Plant Physiology, 121,11691177. 